\title{
ON PRODUCTS IN MINIMAL COMPLEXES
}

\author{
BY \\ J. F. ADAMS
}

1. Let us define an "example-space of the $n$th kind" to be a space with precisely $n$ nonvanishing homotopy groups. Example-spaces of the first kind, then, are those studied by Eilenberg and MacLane ([2], et al.); their singular homology and cohomology groups have been calculated by Cartan [1], and that calculation is of great importance in algebraic topology. It is still a significant problem to calculate the homology and cohomology of examplespaces of higher kinds. The information is needed to develop the theory of the $k$-invariants $\left({ }^{1}\right)$; and by analogy with example-spaces of the first kind, we may expect the higher example-spaces to exemplify cohomology operations of kinds higher than the first.

As a first step in such calculations, J. C. Moore [3] has suggested that products should be introduced in the minimal complexes of such spaces, and has studied such products $\left({ }^{2}\right)$. They are analogous to the products defined by Eilenberg and MacLane [2] in the minimal complexes $\Gamma(\Pi, n)$-products which are fundamental to the work of Eilenberg, MacLane and Cartan.

It is to be noted, however, that it may be proper to give isomorphic minimal complexes different products. For example, define spaces $E_{0}, E_{1}$ so that $\Pi_{r}\left(E_{i}\right)=0(r \neq 2,3), \Pi_{2}\left(E_{i}\right)=Z_{\infty}, \Pi_{3}\left(E_{i}\right)=Z_{2}, k^{4}\left(E_{0}\right)=0, k^{4}\left(E_{1}\right) \neq 0$. Then the minimal complexes $M_{i}$ of the loop-spaces $\Omega\left(E_{i}\right)(i=0,1)$ are isomorphic, being defined by

$$
\Pi_{r} \Omega\left(E_{i}\right)=\Pi_{r+1}\left(E_{i}\right), \quad k^{3} \Omega\left(E_{i}\right)=0 .
$$

However, the Pontrjagin homology products in $\Omega\left(E_{0}\right), \Omega\left(E_{1}\right)$ are different, and to represent this fact, it is proper to define different products in $M_{0}, M_{1}$. We arrive, then, at the notion of products in the minimal complex of an $\mathrm{H}$ space $X$, which are related (in some sense) to the $H$-space product in $X$.

Let $X=\Omega(Y)$ be the space of loops over $Y$ defined by the method due to J. C. Moore (see §2).

THEOREM. If $\Pi_{r}(X)=0$ for $r \neq n, n+1$, and either (i) $n>1$ or (ii) $n=1$ and $k^{8}(X)=0$, then the minimal complex of $X$ admits the structure of a minimal group complex in which the products are A-related to those in $X$.

Received by the editors September 15, 1955.

(1) In the terminology of J. C. Moore, the " $n$th term of a Postnikov system" is such a space.

(2) I am deeply grateful to J. C. Moore for suggesting the present problem to me, and for conversations and letters of help and encouragement. 
The terms "minimal group complex" and " $A$-related" will be defined in $\S 2$.

The existence of an $A$-relation will ensure the existence of the following homotopy-commutative $\left({ }^{3}\right)$ diagram.

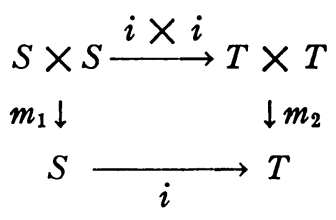

Here $i: S \rightarrow T$ is the injection of the minimal complex $S$ into the total singular complex $T$ of $X . S \times S$ is the Cartesian product of $S$ with $S$ (qua complete semisimplicial complexes); $T \times T$ is similarly defined. $i \times i: S \times S \rightarrow T \times T$ is induced by $i$. The product map $m_{2}$ is induced by the $H$-space product in $X$, and the product map $m_{1}$ is similarly induced by the group-complex product in $S$.

This theorem is by no means final. I conjecture that it would be valid with the following data, where Susp is the suspension homomorphism." " $\Pi_{r}(X)=0$ for $r<n$ and $r>2 n$; $\operatorname{Susp}^{n-r} k^{n+r+1}(X)=0(1 \leqq r \leqq n) . "$

To summarize; the content of the theorem is that certain minimal complexes admit desirable products.

2. Definition of $\Omega(Y)$. Let $Y, y$ be a space with base-point. Let $R$ be the space of real numbers $r \geqq 0$. Let $\bar{\Omega}(Y)$ be the set of loops $\omega:[0,1], 0,1 \rightarrow Y, y, y$ with $C O$-topology; let $\Omega(Y)\left({ }^{4}\right)$ be the set of pairs $(r, \omega)$, where $r \in R$ and $\omega:[0, r], 0, r \rightarrow Y, y, y$ is a loop.

A function $\theta: \Omega(Y) \rightarrow R \times \bar{\Omega}(Y)$ is defined by $\theta(r, \omega)=\left(r, \omega^{\prime}\right)$ where $\omega^{\prime}(t)$ $=\omega(r t) . \theta$ is univalent, and a topology is defined on $\Omega(Y)$ by requiring $\theta$ to be a homeomorphism of $\Omega(Y)$ with its image. It follows easily that $\Omega(Y)$, $\bar{\Omega}(Y)$ are of the same homotopy type.

A continuous map from $\Omega(Y) \times \Omega(Y)$ to $\Omega(Y)$ is defined by $\left(r_{0}, \omega_{0}\right) \cdot\left(r_{1}, \omega_{1}\right)$ $=\left(r_{0}+r_{1}, \omega_{2}\right)$ where

$$
\begin{aligned}
\omega_{2}(t) & =\omega_{0}(t) & \left(0 \leqq t \leqq r_{0}\right), \\
& =\omega_{1}\left(t-r_{0}\right) & \left(r_{0} \leqq t \leqq r_{0}+r_{1}\right) .
\end{aligned}
$$

This product is associative, and has a unit defined by $1=\left(0, \omega_{0}\right)$ where $\omega_{0}(0)=y$.

A group complex $\left({ }^{5}\right) \Gamma$ is a set of groups $\left\{\Gamma_{q}\right\}(q \geqq 0)$. It possesses face and degeneracy operations $\delta_{i}: \Gamma_{q} \rightarrow \Gamma_{q-1}, s_{i}: \Gamma_{q} \rightarrow \Gamma_{q+1}(0 \leqq i \leqq q)$. These are required to be homomorphisms, and to satisfy the $F D$-commutation rules:

(3) "Homotopy" here means the existence of a certain CSS map of the complex $S \times S \times I$, where $I$ is the CSS complex $\mathrm{O}^{\prime}\left(6^{1}\right)$ [2].

(4) These definitions are due to J. C. Moore; but I have taken the liberty of rewording the definition of the topology to be given to $\Omega(Y)$.

(5) This definition is due to J. C. Moore. 


$$
\begin{array}{ll}
\delta_{i} \delta_{j}=\delta_{j-1} \delta_{i} & (i<j), \quad \delta_{j} s_{j} \gamma=\delta_{j+1} s_{j} \gamma=\gamma, \\
s_{i} s_{j}=s_{j+1} s_{i} & (i \leqq j),
\end{array}
$$

Any (geometrical) minimal complex possesses operations $\delta_{i}, s_{i}$, satisfying these rules; but its skeletons do not necessarily have group structure.

We definé( $\left.{ }^{6}\right) \widetilde{\mathrm{II}}_{q}(\Gamma)=\bigcap_{j=0}^{q-1}\left(\delta_{j}^{-1} 1_{q-1}\right)$, where $\delta_{j}: \Gamma_{q} \rightarrow \Gamma_{q-1}$ is the face homomorphism and $1_{q-1}$ the identity of $\Gamma_{q-1}$. A group complex is said to be mini$\operatorname{mal}\left({ }^{6}\right)$ if $\delta_{q}\left(\tilde{\Pi}_{q}\right)=1_{q-1}$ for each $q$.

If a (geometrical) minimal complex $\Gamma$ admits the structure of a group complex (so that the elements of $\Gamma_{q}$ are the minimal $q$-simplexes), then $\Gamma$ is minimal in this sense. For any two elements of $\delta_{q}\left(\tilde{\Pi}_{q}\right)$ are homotopic with boundary fixed, and therefore identical; while $1_{q} \in \tilde{\Pi}_{q}$ and $\delta_{q} 1_{q}=1_{q-1}$.

As above, let the space $X$ be an associative monoid with unit 1 . Let $S$ be a minimal complex of $X$, and suppose products defined in $S^{0}, S^{1}, \cdots, S^{q}$, obeying the axioms for a group complex so far as they are applicable; the unit in $S^{r}$ is required to be the minimal simplex $1_{r}: \sigma^{r} \rightarrow 1$.

The products in $S, X$ are $M$-related if for each pair $f_{1}, f_{2}: \sigma^{r} \rightarrow X$ in $S$ with $r \leqq q$ there is a homotopy $h_{f_{1}, f_{2}}: \sigma^{r} \times I \rightarrow X$ with the following properties.

$$
\begin{aligned}
& h_{f_{1}, f_{2}}(x, 0)=f_{1}(x) \times f_{2}(x) \\
& h_{f_{1}, f_{2}}(x, 1)=\left(f_{1} f_{2}\right) x
\end{aligned}
$$

(product in $X$ ), (product in $S$ ),

(2) $h$ commutes with faces and degeneracies; that is,

$$
h_{\delta_{i} f_{1}, \delta_{i} f_{2}}(x, t)=h_{f_{1}, f_{2}}\left(\delta_{i} x, t\right) \text { and } h_{s_{i} f_{1}, s_{i} f_{2}}(x, t)=h_{f_{1}, f_{2}}\left(s_{i} x, t\right)
$$

whenever both sides are defined. Here $\delta_{i}: \sigma^{r-1} \rightarrow \sigma^{r}$ and $s_{i}: \sigma^{r+1} \rightarrow \sigma^{r}$ are used for the $i$ th face and degeneracy maps.

If either $f_{1}=1_{r}$ or $f_{2}=1_{r}$, then $h_{f_{1}, f_{2}}$ is stationary (independent of $t$ ).

The products in $S, X$ are $A$-related if they are $M$-related, and in addition for each triplet $f_{1}, f_{2}, f_{3}: \sigma^{r} \rightarrow X$ in $S$ with $r \leqq q$ there is a homotopy $H_{f_{1}, f_{2}, f_{3}}$ : $\sigma^{r} \times I \times I \rightarrow X$ commuting with faces $\left({ }^{7}\right)$ such that

$$
\begin{aligned}
& H(x, t, 0)=f_{1}(x) \times f_{2}(x) \times f_{3}(x) \\
& H(x, t, 1)=\left(f_{1} f_{2} f_{3}\right) x \\
& H(x, 0, u)=h_{f_{1}, f_{2}}(x, 2 u) \times f_{3}(x) \\
& H(x, 0, u)=h_{f_{1} f_{2}, f_{3}}(x, 2 u-1) \\
& H(x, 1, u)=f_{1}(x) \times h_{f_{2}, f_{3}}(x, 2 u) \\
& H(x, 1, u)=h_{f_{1}, f_{2} f_{3}}(x, 2 u-1)
\end{aligned}
$$

(product in $X$ ), (product in $S$ ), $(0 \leqq u \leqq 1 / 2)$, $(1 / 2 \leqq u \leqq 1)$, $(0 \leqq u \leqq 1 / 2)$, $(1 / 2 \leqq u \leqq 1)$.

(8) These definitions are all due to J. C. Moore.

$\left({ }^{7}\right)$ Definition as in (2) above. 
3. Lemma 1. If $S^{0} \cdots S^{q-1}$ may be given products $A$-related to those in $X$, then $S^{a}$ may be given a product $M$-related to that in $X$.

Proof. Definition of multiplication. Given any two $q$-simplexes $f_{1}, f_{2}: \sigma^{q} \rightarrow X$ in $S, f_{1} \times f_{2}$ is a singular $q$-simplex of $X$. Its boundary is homotopic to a map $g^{\prime}$ defined by $g^{\prime}\left(\delta_{i} x\right)=\left(\left(\delta_{i} f_{1}\right)\left(\delta_{i} f_{2}\right)\right) x$; a definite homotopy is given by $h^{\prime}\left(\delta_{i} x, t\right)=h_{\delta_{i} f_{1}, \delta_{i} f_{2}}(x, t)$. This homotopy can be extended to a homotopy $h_{f_{1}, f_{2}}$ of $f_{1} \times f_{2}$ with one and only one $q$-simplex $g$ of $S$; we define the product $f_{1} \cdot f_{2}$ to be $g$. This definition makes $\delta_{0} \cdots \delta_{q}$ homomorphisms.

In case $f_{1}=s_{i} f_{1}^{\prime}, f_{2}=s_{i} f_{2}^{\prime}$, we may define the homotopy $h_{f_{1}, f_{2}}$ by the rule

$$
h_{f_{1}, f_{2}}(x, t)=h_{f_{1}, f_{2}^{\prime}}^{\prime}\left(s_{i} x, t\right) ;
$$

this will be consistent. Thus each $s_{i}$ is a homomorphism.

If either $f_{1}=1_{q}$ or $f_{2}=1_{q}$, we may choose $h_{f_{1}, f_{2}}$ to be stationary. Thus $1_{q} f=f 1_{q}=f$. We have thus shown that the product $f_{1} \cdot f_{2}$ is $M$-related to the product in $X$.

Associativity. Take three $q$-simplexes $f_{1}, f_{2}, f_{3}: \sigma^{q} \rightarrow X$ of $s$. We shall define a map $H: \sigma^{q} \times I \times I \rightarrow X$. On $\dot{\sigma}^{q} \times I \times I$ it is defined by

$$
H\left(\delta_{i} x, t, u\right)=H_{\delta_{i} f_{1}, \delta_{i} f_{2}, \delta_{i} f_{3}}(x, t, u) ;
$$

on

$$
\sigma^{q} \times I \times 0, \quad \sigma^{q} \times 0 \times I \text { and } \sigma^{q} \times 1 \times I
$$

it is defined by the equations (4), (6), (7), (8), (9) of $\S 2$; this will be consistent. Therefore we may extend the definition of $H$ throughout $\sigma^{q} \times I \times I$ (without necessarily satisfying (5)). Restricting $H$ to $\sigma^{q} \times I \times 1$, there is a homotopy between $\left(f_{1} \cdot f_{2}\right) \cdot f_{3}$ and $f_{1} \cdot\left(f_{2} \cdot f_{3}\right)$, keeping the boundary fixed. Therefore $\left(f_{1} \cdot f_{2}\right) \cdot f_{3}$ and $f_{1} \cdot\left(f_{2} \cdot f_{3}\right)$ are equal in $S^{q}$.

Inverse elements. Define a homomorphism( $\left(^{8}\right) \theta: S^{q} \rightarrow \sum_{0}^{q} S^{q-1}$ by the rule $\theta f=\left(\delta_{0} f, \delta_{1} f, \cdots, \delta_{q} f\right)$. We first show that $\theta\left(S^{q}\right)$ contains inverse elements. Let $f \in S^{q}$, and take $\theta f$, that is $\left(\delta_{0} f, \delta_{1} f, \cdots, \delta_{q} f\right)$. There is a $g$ in $S^{q}$ such that $\delta_{i} g=\left(\delta_{i} f\right)^{-1}(0 \leqq i \leqq q-1)$; for these conditions are consistent, and $S$ is a minimal complex. Thus $\theta(f \cdot g)=\left(1,1, \ldots, 1, \delta_{q} f \cdot \delta_{q} g\right)$. Since $S$ is a minimal complex, $\delta_{q} f \cdot \delta_{q} g=1$, and $\theta(f \cdot g)=1$. Similarly $\theta(g \cdot f)=1$.

The kernel $K^{q}$ of $\theta$ is a subset of $S^{q}$ consisting of $q$-simplexes whose $(q-1)$-faces are all $1_{q-1}$. This, with the product given to it, is isomorphic to $\Pi_{q}(X)$, and certainly contains inverses. The existence of left and right inverses in $S^{q}$ follows; given $f \in S^{q}$, there is a $g \in S^{q}$ with $f g=k_{1}, g f=k_{2}$ both in $K^{q}$; therefore, using the associative law, $f\left(g k_{1}^{-1}\right)=1_{q},\left(k_{2}^{-1} g\right) f=1_{q}$.

4. Let us revert to the situation of the previous paragraph, headed "Associativity" (in §3). Since we now know that $\left(f_{1} \cdot f_{2}\right) \cdot f_{3}=f_{1} \cdot\left(f_{2} \cdot f_{3}\right)$, we may $S^{a-1}$.

(8) The following formula stands for the direct sum of $(q+1)$ groups each isomorphic with 
define $H$ on $\sigma^{q} \times I \times 1$ by equation (5), $\S 2$. And if $\Pi_{q+1}(X)=0$, we may extend the definition of $H$ over $\sigma^{q} \times I \times I$, and so obtain an $A$-relation between the products in $S^{q}$ and $X$. This remark will allow us to prove the theorem by induction (starting from the trivial $A$-related products in $S^{0} \cdots S^{n-1}$ ) once we have considered the obstruction to an $A$-relation in dimension $q=n$. Accordingly, it only remains to consider this obstruction, and show that it is zero.

The obstruction consists of an element of $\Pi_{n+1}(X)$ for each triplet $\left(f_{1}, f_{2}, f_{3}\right)$ of elements of $S^{n}$. As we have noted, $S^{n}$ is isomorphic with $\Pi_{n}(X)$; we interpret the obstruction as a 3-cochain $c^{3}$ of $\Pi_{n}(X)$ in $\Pi_{n+1}(X)$. By construction, $c^{3}$ is zero on triplets for which one of $f_{1}, f_{2}, f_{3}$ is 1 .

We are entitled to change the homotopy $h$ constructed by Lemma 1 , and such a change will in general change $c^{3}$. That is to say, we may change $h_{f_{1}, f_{2}}$ through $c^{2}\left(f_{1}, f_{2}\right)$ where $c^{2}$ is any 2-cochain of $\Pi_{n}(X)$ in $\Pi_{n+1}(X)$, provided that $c^{2}\left(f_{1}, f_{2}\right)=0$ if either $f_{1}$ or $f_{2}$ is 1 . The resulting change in $c^{3}\left(f_{1}, f_{2}, f_{3}\right)$ is, to within a sign,

$$
c^{2}\left(f_{2}, f_{3}\right)+c^{2}\left(f_{1}, f_{2} f_{3}\right)-c^{2}\left(f_{1} f_{2}, f_{3}\right)-c^{2}\left(f_{1}, f_{2}\right)=\delta c^{2} .
$$

We conclude that the obstruction to an $A$-relation in dimension $q=n$ is a coset $\left\{c^{3}\right\}$ of $B^{3}\left(\Pi_{n} ; \Pi_{n+1}\right)$ in $C^{3}\left(\Pi_{n} ; \Pi_{n+1}\right)$; and if this coset is zero, the theorem is proved.

5. To identify $\left\{c^{3}\right\}$, consider $Z$, the space of maps of an $(n-1)$-element $e^{n-1}, \dot{e}^{n-1} \rightarrow X, 1$ (or $Z=X$, if $n=1$ ). The product in $X$ induces one in $Z$, by the rule $(f \times g) p=(f p) \times(g p)\left(p \in e^{n-1}\right)$. We write 1 for the unit in $Z$. We have $\Pi_{1}(Z)=\Pi_{n}(X), \Pi_{2}(Z)=\Pi_{n+1}(X)$.

LEMMA 2. $\left\{c^{3}\right\}=k^{3}(Z)$.

This will clearly conclude the argument in case $n=1$.

Proof. It is trivial to translate the previous constructions into $Z$. We find in $Z$ the following maps.

(a) For each element $\alpha$ of $\Pi_{1}(Z)$, a map $f_{\alpha}: I, \dot{I} \rightarrow Z, 1$ representing $\alpha$.

(b) For each pair $(\alpha, \beta)$ of elements of $\Pi_{1}(Z)$, a homotopy $h_{\alpha, \beta}: I^{2}, \dot{I} \times I$ $\rightarrow Z, 1$ such that $h_{\alpha, \beta}(x, 0)=f_{\alpha}(x) \times f_{\beta}(x), h_{\alpha, \beta}(x, 1)=f_{\alpha \beta}(x)$.

For each triplet $(\alpha, \beta, \gamma)$ of elements of $\Pi_{1}(Z)$ a map $H_{\alpha, \beta, \gamma}: \dot{I}^{3} \rightarrow Z$ defined by the following equations.

$$
\begin{array}{rlr}
H_{\alpha, \beta, \gamma}(x, t, u) & =1 & (x=0 \text { or } 1) . \\
H_{\alpha, \beta, \gamma}(x, t, u) & =f_{\alpha}(x) \times f_{\beta}(x) \times f_{\gamma}(x) & \\
& (0 \leqq t \leqq 1 / 2 \& u=0 ; \text { also } t=0 \& 0 \leqq u \leqq 1 / 2) . \\
H_{\alpha, \beta, \gamma}(x, 0, u) & =h_{\alpha, \beta}(x, 2 u-1) \times f_{\gamma}(x) & (1 / 2 \leqq u \leqq 1) . \\
H_{\alpha, \beta, \gamma}(x, 1, u) & =h_{\alpha, \beta \gamma}(x, u) .
\end{array}
$$




$$
\begin{aligned}
& H_{\alpha, \beta, \gamma}(x, t, 0)=f_{\alpha}(x) \times h_{\beta, \gamma}(x, 2 t-1) \quad(1 / 2 \leqq t \leqq 1) . \\
& H_{\alpha, \beta, \gamma}(x, t, 1)=h_{\alpha \beta, \gamma}(x, t) .
\end{aligned}
$$

(We have here made a trivial alteration to $H$ for later convenience.) The element of $\Pi_{2}(Z)$ represented by $H_{\alpha, \beta, \gamma}$ is $c^{3}(\alpha, \beta, \gamma)$.

The proof proceeds by constructing a homotopy $H_{\alpha, \beta, \gamma}^{1}: \dot{I}^{3} \times I \rightarrow Z$ such that $H_{\alpha, \beta, \gamma}^{1} \mid \dot{I}^{3} \times 1=H_{\alpha, \beta, \gamma}$, and $H_{\alpha, \beta, \gamma}^{1} \mid \dot{I}^{3} \times 0=H_{\alpha, \beta, \gamma}^{2} m$. Here $m: \dot{I}^{3} \rightarrow \dot{\sigma}^{3}$ is a generator of $\Pi_{2}\left(\dot{\sigma}^{3}\right)$, and $H_{\alpha, \beta, \gamma}^{2}: \dot{\sigma}^{3} \rightarrow Z$ has the following faces.

$$
\delta_{0} H^{2}=h_{\beta, \gamma}^{3}, \quad \delta_{1} H^{2}=h_{\alpha \beta, \gamma}^{3}, \quad \delta_{2} H^{2}=h_{\alpha, \beta \gamma}^{3}, \quad \delta_{3} H^{2}=h_{\alpha, \beta}^{3} .
$$

Here, for each pair $(\alpha, \beta)$ of elements of $\Pi_{1}(Z), h_{\alpha, \beta}^{3}: \sigma^{2} \rightarrow Z$ is a map with the following faces.

$$
\delta_{0} h^{3}=f_{\beta}, \quad \delta_{1} h^{3}=f_{\alpha \beta}, \quad \delta_{2} h^{3}=f_{\alpha} .
$$

It will evidently follow from this that the cochain $c^{3}(\alpha, \beta, \gamma)$ represents $k^{3}(Z)$.

We perform the construction as follows.

Define $h_{\alpha, \beta}^{1}(x, t)=f_{\alpha} \theta_{1}(x, t) \times f_{\beta} \theta_{2}(x, t): I^{2}, \dot{I} \times I \rightarrow Z, 1$. Here the auxiliary functions $\theta_{1}, \theta_{2}: I^{2} \rightarrow I$ are defined as follows. On $\dot{I}^{2}$ they satisfy the following conditions.

$$
\begin{array}{lrl}
\theta_{i}(x, t) & =x & (x=0,1 ; i=1,2) . \\
\theta_{i}(x, 1)=x . & & (0 \leqq x \leqq 1 / 2) . \\
\theta_{1}(x, 0)=2 x, \theta_{2}(x, 0)=0 & & (1 / 2 \leqq x \leqq 1) . \\
\theta_{1}(x, 0)=1, \theta_{2}(x, 0)=2 x-1 &
\end{array}
$$

Since the space $I$ is contractible, $\theta_{i}$ may be extended over $I^{2}$; we shall suppose this done in a fixed way. Define

$$
\begin{aligned}
h_{\alpha, \beta}^{2}(x, t) & =h_{\alpha, \beta}^{1}(x, 2 t) & & (0 \leqq t \leqq 1 / 2) \\
& =h_{\alpha, \beta}(x, 2 t-1) & & (1 / 2 \leqq t \leqq 1) .
\end{aligned}
$$

Define $l: I^{2} \rightarrow \sigma^{2}$ so that it gives a homeomorphism between the interiors of $I^{2}, \sigma^{2}$, and satisfies the following conditions on $\dot{I}^{2}$.

$$
\begin{array}{rlrl}
l(0, t) & =(1,0,0), & \\
l(1, t) & =(0,0,1), & & \\
l(x, 0) & =(1-2 x, 2 x, 0) & & (0 \leqq x \leqq 1 / 2) \\
& =(0,2-2 x, 2 x-1) & & (1 / 2 \leqq x \leqq 1), \\
l(x, 1) & =(1-x, 0, x) . & &
\end{array}
$$

Define $h_{\alpha, \beta}^{3}: \sigma^{2} \rightarrow Z$ by the rule $h_{\alpha, \beta}^{2}=h_{\alpha, \beta}^{3} l$. By construction, $h_{\alpha, \beta}^{3}$ has the faces required. 
For future use, we define $L=l(I \times[0,1 / 2])$. The maps $\theta_{i}^{\prime}: L \rightarrow I$ defined by $\theta_{i}^{\prime} l(x, t)=\theta_{i}(x, 2 t)(i=1,2)$ are continuous. Define $m: \dot{I}^{3} \rightarrow \dot{\sigma}^{3}$ as follows:

$$
\begin{array}{rlr}
m\left(0 \times I^{2}\right) & =(1000) . m\left(1 \times I^{2}\right)=(0001) \\
m \mid I \times 1 \times I & =l: I^{2} \rightarrow \delta_{2} \sigma^{3} . & \\
m \mid I \times I \times 1 & =l: I^{2} \rightarrow \delta_{1} \sigma^{3} . & \\
m(x, 0, u) & =l(2 x, u): I^{2} \rightarrow \delta_{2} \sigma^{3} & (0 \leqq x \leqq 1 / 2,1 / 2 \leqq u \leqq 1) \\
& =(0,0,2-2 x, 2 x-1) & (1 / 2 \leqq x \leqq 1,1 / 2 \leqq u \leqq 1) . \\
m(x, t, 0) & =(1-2 x, 2 x, 0,0) & (0 \leqq x \leqq 1 / 2,1 / 2 \leqq t \leqq 1) \\
& =l(2 x-1, t): I^{2} \rightarrow \delta_{0} \sigma^{3} & (1 / 2 \leqq x \leqq 1,1 / 2 \leqq t \leqq 1) . \\
m(x, 0,0) & =(1-3 x, 3 x, 0,0) & (0 \leqq x \leqq 1 / 3) \\
& =(0,2-3 x, 3 x-1,0) & (1 / 3 \leqq x \leqq 2 / 3) \\
& =(0,0,3-3 x, 3 x-2) & (2 / 3 \leqq x \leqq 1) . \\
m(x, 0, u) & \in A & (0 \leqq u \leqq 1 / 2) . \\
m(x, t, 0) & \in B & (0 \leqq t \leqq 1 / 2) .
\end{array}
$$

Here the set $A$ is the union of $\delta_{0} \delta_{0} \sigma^{3}$ with the part $L$ of $\delta_{3} \sigma^{3}$, and the set $B$ is the union of $\delta_{2} \delta_{3} \sigma^{3}$ with the part $L$ of $\delta_{0} \sigma^{3}$. Since $A$ and $B$ are contractible, the definition of $m$ may be extended over $\dot{I}^{3}$.

Define $H_{\alpha, \beta, \gamma}^{1}: \dot{I}^{3} \times I \rightarrow Z$ as follows:

$$
\begin{array}{rrr}
H_{\alpha, \beta, \gamma}^{1}(x, t, u, v) & =1 & (x=0 \text { or } 1) . \\
H_{\alpha, \beta, \gamma}^{1}(x, t, u, v) & =f_{\alpha} \psi_{1}(x, t, u, v) \times f_{\beta} \psi_{2}(x, t, u, v) \times f_{\gamma} \psi_{3}(x, t, u, v) \\
\quad(0 \leqq t \leqq 1 / 2 \& u=0 ; \text { also } t=0 \& 0 \leqq u \leqq 1 / 2) . & \\
H_{\alpha, \beta, \gamma}^{1}(x, 0, u, v) & =h_{\alpha, \beta}\left(\theta_{1}(x, v), 2 u-1\right) \times f_{\gamma} \theta_{2}(x, v) & (1 / 2 \leqq u \leqq 1) . \\
H_{\alpha, \beta, \gamma}^{1}(x, t, 0, v) & =f_{\alpha} \theta_{1}(x, v) \times h_{\beta, \gamma}\left(\theta_{2}(x, v), 2 t-1\right) & (1 / 2 \leqq t \leqq 1) . \\
H_{\alpha, \beta, \gamma}^{1}(x, 1, u, v) & =h_{\alpha, \beta \gamma}^{2}(x, \phi(u, v)) . \\
H_{\alpha, \beta, \gamma}^{1}(x, t, 1, v) & =h_{\alpha \beta, \gamma}^{2}(x, \phi(t, v)) .
\end{array}
$$

The subsidiary functions are defined as follows. The map $\phi: I^{2} \rightarrow I$ satisfies the following conditions on $\dot{I}^{2}$.

$$
\begin{array}{ll}
\phi(0, v)=v / 2, & \phi(1, v)=1, \\
\phi(t, 0)=t, & \phi(t, 1)=(t+1) / 2 .
\end{array}
$$

Since $I$ is contractible, $\phi$ may be extended over $I^{2}$.

The maps $\psi_{i}(i=1,2,3)$ into $I$ shall satisfy the following conditions.

$$
\begin{aligned}
& \psi_{i}(x, t, u, v)=x \\
& (x=0,1) \text {. } \\
& \psi_{i}(x, t, u, v)=x \\
& (0 \leqq t \leqq 1 / 2 \& u=0 \text {; also } t=0 \& 0 \leqq u \leqq 1 / 2) \text {. }
\end{aligned}
$$




$$
\begin{array}{c|c|c|c}
\frac{\psi_{1}(x, t, u, v)=}{\theta_{1}(x, v)} & \frac{\psi_{2}(x, t, u, v)=}{\theta_{1}(x, v)} & \frac{\psi_{3}(x, t, u, v)=}{\theta_{2}(x, v)} & (t=0, u=1 / 2) \\
\theta_{1}(x, v) & \theta_{2}(x, v) & \theta_{2}(x, v) & (t=1 / 2, u=0) \\
\theta_{1}^{\prime} \chi_{2} m(x, t, u) & \theta_{2}^{\prime} \chi_{2} m(x, t, u) & \chi_{3} m(x, t, u) & (t=0,0 \leqq u \leqq 1 / 2, v=0) \\
\chi_{0} m(x, t, u) & \theta_{1}^{\prime} \chi_{1} m(x, t, u) & \theta_{2}^{\prime} \chi_{1} m(x, t, u) & (0 \leqq t \leqq 1 / 2, u=0, v=0) .
\end{array}
$$

Since $I$ is contractible, each map $\psi_{i}$ may be extended over its domain of definition. The subsidiary functions are defined as follows.

$$
\begin{aligned}
\chi_{0}\left(y_{0}, y_{1}, y_{2}, y_{3}\right) & =y_{1} \text { on } \delta_{2} \delta_{3} \sigma^{3} \\
& =1 \text { on } \delta_{0} \sigma^{3}, \\
\chi_{1}\left(y_{0}, y_{1}, y_{2}, y_{3}\right) & =\left(y_{1}, y_{2}, y_{3}\right) \text { on } \delta_{0} \sigma^{3} \\
& =(1,0,0) \text { on } \delta_{2} \delta_{3} \sigma^{3}, \\
\chi_{2}\left(y_{0}, y_{1}, y_{2}, y_{3}\right) & =\left(y_{0}, y_{1}, y_{2}\right) \text { on } \delta_{3} \sigma^{3} \\
& =(0,0,1) \text { on } \delta_{0} \delta_{0} \sigma^{3}, \\
\chi_{3}\left(y_{0}, y_{1}, y_{2}, y_{3}\right) & =y_{3} \text { on } \delta_{0} \delta_{0} \sigma^{3} \\
& =0 \text { on } \delta_{3} \sigma^{3} .
\end{aligned}
$$

These constructions, with the usual verifications, conclude the proof of Lemma 2.

6. Lemma 3. If $n>1, k^{3}(Z)=0$.

Proof. We first recall that the $k$-invariants of a loop-space are given by $\left({ }^{9}\right)$

$$
k^{q}(\bar{\Omega}(W))=-\operatorname{Susp} k^{q+1}(W) .
$$

Using any homeomorphism between $e^{n-1}, \dot{e}^{n-1}$ and $I^{n-1}, \dot{I}^{n-1}$ we find a homeomorphism between $Z$ and $\bar{\Omega}^{n-1}(X)$. Thus $k^{3}(Z)=(-1)^{n} \operatorname{Susp}^{n} k^{n+8}(Y)(n \geqq 2)$. Write $\Pi=\Pi_{n+1}(Y), \Pi^{\prime}=\Pi_{n+2}(Y)$. Choose a base of ${ }_{2}\left(\Pi^{\prime}\right)$, and construct from it a symmetric idempotent bilinear pairing of ${ }_{2}\left(\Pi^{\prime}\right)$ to itself [4]. For $n \geqq 2$, we have an isomorphism $[1$, p. 707]

$$
\theta: H_{n+3}\left(\Pi, n+1 ; Z_{\infty}\right) \cong(\Pi)_{2} \text {. }
$$

Therefore $H^{n+3}\left(\Pi, n+1, \Pi^{\prime}\right) \cong$ Hom $\left((\Pi)_{2}, \Pi^{\prime}\right)$. Let the image of $k^{n+3}$ be the homomorphism $\eta:(\Pi)_{2} \rightarrow_{2}\left(\Pi^{\prime}\right)$. Appealing to the definition of $\theta$, we have $k^{n+3}(Y)=S q^{2}\left\{\eta^{\prime}\right\}$, where $S q^{2}: H^{n+1}\left(Y ;{ }_{2}\left(\Pi^{\prime}\right)\right) \rightarrow H^{n+3}\left(Y ;{ }_{2}\left(\Pi^{\prime}\right)\right)$ is the Steenrod square, defined using the pairing above, and $\left\{\eta^{\prime}\right\}$ is the class of the homomorphism $\eta^{\prime}: \Pi \rightarrow_{2}\left(\Pi^{\prime}\right)$ induced by $\eta$. Since $S q^{2}$ and Susp commute, we have $k^{3}(z)=(-1)^{n} S q^{2}\left\{\eta^{\prime}\right\}$, where $\left\{\eta^{\prime}\right\} \in H^{1}\left(Z ;{ }_{2}\left(\Pi_{2}(Z)\right)\right)$ is the class of $\eta^{\prime}: \Pi_{1}(Z)$

(9) This theorem appears to be known, although I do not know to whom it should be ascribed. I am in possession of a proof. 
$\rightarrow_{2}\left(\Pi_{2}(Z)\right)$. Since $S q^{2} h=0$ for any 1 -class $h$, we have $k^{3}(z)=0$. This concludes the proof of Lemma 3 , and of the main theorem.

7. Existence of a non-Abelian group complex. Some general remarks are first necessary. Let $\Gamma$ be a group complex. We may distinguish a subcomplex $K$ consisting of $1_{r}(0 \leqq r \leqq n)$ together with all elements $\sigma$ of $\Gamma_{m}(m>n)$ such that each $n$-face $\Delta_{I} \sigma$ is the identity $1_{n}$. (Here $\Delta_{I}=\delta_{i_{1}} \delta_{i_{2}} \cdots \delta_{i_{m-n}}$ ). $K$ is closed under $\delta_{i}, s_{j}$ and the product; thus it is a group complex embedded in $\Gamma . K$ is normal; for if $\sigma \in \Gamma, \tau \in K, \Delta_{I}\left(\sigma \tau \sigma^{-1}\right)=\left(\Delta_{I} \sigma\right)\left(\Delta_{I} \sigma\right)^{-1}=1_{n}$. We may thus form the quotient $\Gamma / K=L$; this is again a group complex. If $\Gamma$ is minimal, $K$ and $L$ are minimal.

We recall that $\Pi_{n}(\Gamma)$ is written for $\operatorname{Ker} \delta_{q} / \operatorname{Im} \delta_{q+1}$, where $\delta_{q}: \tilde{\Pi}_{q}(\Gamma)$ $\rightarrow \tilde{\Pi}_{q-1}(\Gamma)$ and $\delta_{q+1}: \tilde{\Pi}_{q+1}(\Gamma) \rightarrow \tilde{\Pi}_{q}(\Gamma)$ are the boundary maps. In case $\Gamma$ is minimal, $\Pi_{q}(\Gamma)=\tilde{\Pi}_{q}(\Gamma)$. In case $\Gamma$ is the minimal complex of a space $X$, $\Pi_{n}(\Gamma) \cong \Pi_{n}(X)$, whatever product (consistent with the axioms) be given to $\Gamma$. For any quotient $\Gamma / K=L$ of group complexes, we have an exact homotopy sequence $\rightarrow \Pi_{n}(K) \rightarrow \Pi_{n}(\Gamma) \rightarrow \Pi_{n}(L) \rightarrow \Pi_{n-1}(K) \rightarrow$. In the case constructed above, we have

$$
\begin{aligned}
& \Pi_{r}(K)=0, \\
& \Pi_{r}(L) \cong \Pi_{r}(\Gamma) \\
& (r \leqq n) \\
& \cong \pi_{r}(\Gamma), \\
& =0 \text {, } \\
& (r>n) \text {. }
\end{aligned}
$$

The construction is of course intended as an analogue of the process of "killing homotopy groups."

To construct a non-Abelian group complex, it is sufficient to arrange a non-Abelian group extension of two Eilenberg-MacLane complexes. We may choose to construct a central extension, with $K=\Gamma\left(Z_{\infty}, 1\right)$ and $L=\Gamma\left(Z_{2}, 2\right)$. It is sufficient to construct the extension up to $\Gamma_{3}$, by the following lemma.

LemMa 4. Let $\Gamma_{r}$, for $r<k$, obey the axioms for a group complex, so far as they are applicable. Then there is a group $\Gamma_{k}$, and homomorphisms $s_{i}: \Gamma_{k-1} \rightarrow \Gamma_{k}$, $\delta_{i}: \Gamma_{k} \rightarrow \Gamma_{k-1}$, obeying the axioms for a group complex so far as they are applicable, and such that the sequence

$$
0 \rightarrow \widetilde{\Pi}_{k} \stackrel{\delta}{\rightarrow} \Pi_{k-1} \stackrel{\delta}{\rightarrow} \widetilde{\Pi}_{k-2}
$$

is exact.

The lemma is not hard to prove.

In our case, if $\Gamma_{3}$ is constructed, we may embed it in a minimal group complex $\Gamma$ by applying Lemma 4 inductively. $\Gamma$ will have $\Pi_{r}(\Gamma)=0(r>2)$; thus the kernel $K$ of $\Gamma \rightarrow \Gamma\left(Z_{\infty}, 1\right)$ will have $\Pi_{r}(K)=0(r>2)$; by Moore's results $K$ must be an Eilenberg-MacLane complex, necessarily $\Gamma\left(Z_{1}, 2\right)$.

It remains to describe $\Gamma_{2}, \Gamma_{3}$. Let $a, b$ be elements of dimensions 1,2 , generating $\Gamma_{1}, K_{2}$. As one may verify, the formula $\left(s_{0} a\right)\left(s_{1} a\right)\left(s_{0} a\right)^{-1}\left(s_{1} a\right)^{-1}=b$ defines (consistently) the central group extensions $\Gamma_{2}, \Gamma_{3}$. 
One may also compute the Pontrjagin homology square of the generator of $H_{1}(\Gamma)$, and verify that it agrees with its analogue in the space $\Omega\left(E_{1}\right)$.

\section{REFERENCES}

1. H. Cartan, Sur les groupes d'Eilenberg-MacLane $H(\Pi, n)$, Proc. Nat. Acad. Sci. U.S.A. vol. 40 (1954) pp. 467-471, 704-707.

2. S. Eilenberg and S. MacLane, On the groups $H(I I, n)$ I, II, Ann. of Math. vol. 58 (1953) pp. 55-106, and vol. 60 (1954) pp. 49-139.

3. J. C. Moore, Séminaire H. Cartan. E.N.S., 1954-1955, Exp. 18, 19.

4. J. H. C. Whitehead, On the theory of obstructions, Ann. of Math. vol. 54 (1951) p. 77.

OXFord University,

OXFORD, ENGLAND. 